\title{
New Composite Operations and Precomputation Scheme for Elliptic Curve Cryptosystems over Prime Fields
}

\author{
Patrick Longa ${ }^{1}$ and Ali Miri ${ }^{2}$ \\ ${ }^{1}$ Department of Electrical and Computer Engineering \\ University of Waterloo, Canada \\ plonga@uwaterloo.ca \\ ${ }^{2}$ School of Information Technology and Engineering (SITE) \\ University of Ottawa, Canada \\ Ali.Miri@uottawa.ca
}

\begin{abstract}
We present a new methodology to derive faster composite operations of the form $d P+Q$, where $d$ is a small integer $\geq 2$, for generic ECC scalar multiplications over prime fields. In particular, we present an efficient Doubling-Addition (DA) operation that can be exploited to accelerate most scalar multiplication methods, including multiscalar variants. We also present a new precomputation scheme useful for window-based scalar multiplication that is shown to achieve the lowest cost among all known methods using only one inversion. In comparison to the remaining approaches that use none or several inversions, our scheme offers higher performance for most common $I / M$ ratios. By combining the benefits of our precomputation scheme and the new DA operation, we can save up to $6.2 \%$ on the scalar multiplication using fractional $w \mathrm{NAF}$.
\end{abstract}

Keywords: Elliptic curve cryptosystem, scalar multiplication, point operation, composite operation, precomputation scheme.

\section{Introduction}

Elliptic curve cryptography (ECC) was independently introduced by Koblitz and Miller in 1985. Since then, this public-key cryptosystem has attracted increasing attention due to its shorter key size requirement in comparison with other established systems such as RSA and DL-based cryptosystems. For instance, it is widely accepted that 160-bit ECC offers equivalent security to 1024-bit RSA. This significant difference makes ECC especially attractive for applications in constrained environments as shorter key sizes are translated to less power and storage requirements, and reduced computing times.

Scalar multiplication, denoted by $k P$, where $k$ is the secret key (scalar) and $P$ is a point on the elliptic curve, is the central operation of elliptic curve cryptosystems. Methods to efficiently compute such operation have traditionally exploited the binary expansion of numbers (e.g., NAF and wNAF). This is mainly due to 
the fact that the binary expansion directly translates to computations using the simplest elementary ECC point operations, namely point doubling and addition.

However, recent developments in the field suggest that it is possible to use more complex operations to accelerate the scalar multiplication 4/7/8/24. For instance, Ciet et al [4] introduced the ternary/binary method using radices 2 and 3 for the representation of the scalar. Dimitrov et al [7] proposed the doublebase number system for the scalar multiplication using mixed powers of 2 and 3. Radix 5 was added to the previous approach by Mishra et al 21] to represent scalars with mixed powers of 2, 3 and 5. More recently, Longa and Miri [18 have proposed the multibase non-adjacent form $(\mathrm{mbNAF})$ method, which uses a very efficient representation of integers using multiple bases. Efficiency of the previous methods strongly depends on the costs of such operations as tripling $(3 P$, denoted by $T$ ) or quintupling $(5 P$, denoted by $Q)$ of a point, unified doublingaddition $(2 P+Q$, denoted by $\mathrm{DA})$, unified tripling-addition $(3 P+Q$, denoted by TA), unified quintupling-addition $(5 P+Q$, denoted by $\mathrm{QA})$, among others. Thus, it is a critical task to reduce the computing cost of these operations, which are referred to as composite operations since they are inherently based on basic doubling and addition, to further speed up the execution time of the scalar multiplication.

In the first part, we propose a new technique to derive faster composite operations of the form $d P+Q$, where $d$ is a small integer $\geq 2$ and $P, Q$ are points on the elliptic curve in Jacobian and affine coordinates, respectively. As pointed out, operations of this form are highly common in all known scalar multiplication methods, including multiscalar versions which are used in the ECDSA signature verification [12. For instance, DA is a recurrent operation in NAF, $w$ NAF and Shamir's trick, where each mixed Jacobian-affine addition is always computed right after a doubling. In addition to DA, TA is used in the doublebase method [7], and TA and QA in the triple-base method [21] and $m b \mathrm{NAF}$ methods [1618.

Our technique makes use of Meloni's idea of adding two points with the same $z$ coordinates [19] (which we will refer to as special addition with identical $z$ coordinate) to have an iterative computation of the form $d P+Q=P+\ldots+$ $P+P+(P+Q)$, which is computed backwards and where only the first addition shown in parentheses is computed with a traditional mixed addition. Every extra addition can then be efficiently computed with the addition with identical $z$ coordinate. We show that our new composite operations are more efficient than formulas using the cheapest operations existent in current literature. See [17] for the state-of-the-art point formulas in Jacobian coordinates.

In the second part, we modify the previous methodology to yield a new scheme for the precomputation of points in window-based methods such as $w \mathrm{NAF}$ and fractional $w \mathrm{NAF}$ (denoted by Frac- $w \mathrm{NAF}$ ). Using pre-stored points is a practical technique to accelerate the scalar multiplication when there is extra memory available. However, for scalar multiplications $k P$ with $P$ unknown, the precomputation of such points is necessarily done on-line and its time execution included in the whole time estimation of the scalar multiplication. Examples of this case 
can be found during decryption in the ElGamal encryption scheme or in the Diffie-Hellman key exchange. Thus, it is crucial to reduce the time for the precomputation to boost the savings achieved by window-based methods.

Given that precomputations follow the form $d_{i} P$, where $d_{i}$ are the odd integers in the range $[3, m]$ with $m \geq 3$, we modify our original approach to the iterative computation of the form $d_{i} P=2 P+\ldots+2 P+2 P+2 P+P$, which is again computed backwards, and requires one point doubling followed by cheaper additions with identical $z$-coordinate. Following, and to keep advantage of the efficient mixed addition during the scalar multiplication, points are converted to affine representation using the well-known Montgomery's method which permits to reduce the number of expensive inversions to only one. Moreover, this method is further sped up by efficiently using values computed during the first stage of our methodology. For the latter, two variants with different memory requirements are presented, which will be shown to be suitable for different window widths.

Our precomputation scheme is compared with the best previous approaches using only one inversion, and shown to deliver the lowest cost. In comparison to methods using none or several inversions, our scheme is shown to offer the highest performance for most common $I / M$ ratios.

Our work is organized as follows. In Section 2, we detail some background about ECC over prime fields. Then, we present our methodology based on the special addition with identical $z$-coordinate, and apply it to derive efficient composite operations of the form $d P+Q$, whose costs are compared to previous formulae right after. In Section 4, a variant of the previous methodology is used to build a new precomputation scheme for window-based scalar multiplications. The cost and memory requirements of our scheme are then discussed and compared with previous efforts. Some conclusions summarizing the contributions of this work are presented at the end.

\section{Preliminaries}

An elliptic curve $E$ over a prime field $\mathbb{F}_{p}$ (denoted by $E\left(\mathbb{F}_{p}\right)$ ) is defined by the reduced Weierstrass equation [12]:

$$
E: y^{2}=x^{3}+a x+b .
$$

Where: $a, b \in \mathbb{F}_{p}$ and $\triangle=4 a^{3}+27 b^{2} \neq 0$.

The set of pairs $(x, y)$ that solves (1), where $x, y \in \mathbb{F}_{p}$, and the point at infinity $\mathcal{O}$, which is the identify for the group law, form an abelian group $\left(E\left(\mathbb{F}_{p}\right),+\right)$, over which the ECC computations are performed.

The main operation in ECC is known as scalar multiplication, which is denoted by $Q=k P$, where $P$ and $Q$ are points in $E\left(\mathbb{F}_{p}\right)$, and $k$ is the secret scalar.

The simplest representation of points on the elliptic curve $E$ with two coordinates $(x, y)$, namely affine coordinates (denoted by $\mathcal{A}$ ), introduces field inversions into the computation of point doubling and addition. Inversions over prime fields are the most expensive field operation and are avoided as much as possible. 
Although their relative cost depends on the characteristics of a particular implementation, it has been observed that, especially in the case of efficient forms for the prime $p$ as recommended by 11, the inversion can result as computationally expensive as $1 I>30 M$. For instance, benchmarks presented by 15 and 212 show $I / M$ ratios between $30-40$ and 50-100, respectively.

Projective coordinates $(X, Y, Z)$ solve the previous problem by adding the third coordinate $Z$ to replace inversions with a few other field operations. The foundation of these inversion-free coordinate systems can be explained by the concept of equivalence class, which is defined in the following in the context of Jacobian coordinates $\mathcal{J}$, a special case of projective coordinates that has yielded very efficient point formulae 10 .

Given a prime field $\mathbb{F}_{p}$, there is an equivalence relation $\equiv$ among non-zero triplets over $\mathbb{F}_{p}$, such that [1]:

$\left(X_{1}, Y_{1}, Z_{1}\right) \equiv\left(X_{2}, Y_{2}, Z_{2}\right) \Leftrightarrow X_{1}=\lambda^{2} X_{2}, Y_{1}=\lambda^{3} Y_{2}$ and $Z_{1}=\lambda Z_{2}$, for some $\lambda \in \mathbb{F}_{p}^{*}$.

Thus, the equivalence class of a (Jacobian) projective point, denoted by $(X: Y: Z)$, is:

$$
\left.(X, Y, Z)=\left\{\lambda^{2} X, \lambda^{3} Y, \lambda Z\right): \lambda \in \mathbb{F}_{p}^{*}\right\} .
$$

It is important to remark that any $(X, Y, Z)$ in the equivalence class (2) can be used as a representative of a given projective (Jacobian) point.

In the following, we succinctly summarize costs of the improved formulae in $\mathcal{J}$ introduced by [17, which applied an effective technique to speed up the traditional point operations. The improved formulae will be later used for comparison with our new composite operations in Section 3. For further details about point formulae the reader is referred to 17 .

The cost of using the Jacobian representation for the doubling formula has been found to be $2 M+8 S$ (reduced from the traditional $4 M+6 S$ ). When $w$ successive executions of several doublings are used, the cost is $(3 w) M+(5 w+2) S$ by combining the strategy in 17 to accelerate operations with the approach by [14, which means that doublings are performed with only $3 M+5 S$, with exception of the first one that costs $3 M+7 S$.

Also, it is important to note that it has been suggested that the parameter $a$ (see (1)) be fixed at -3 for efficiency purposes. In fact, most curves recommended by public-key standards [13] use $a=-3$, which has been shown to not impose significant restrictions to the cryptosystem [3]. In this case, the cost of point doubling is reduced to only $3 M+5 S$.

In the remainder of this work, we will refer to the special case when $a=-3$, and the general case when the parameter is not fixed and can be any value in the field.

In the case of addition, representing one of the points in $\mathcal{J}$ and the other in $\mathcal{A}$ has yielded the most efficient addition formula, which is known as mixed Jacobian-affine addition and presents a cost of $8 M+3 S$ [5]. In [17, the cost of 
this operation was reduced further to only $7 M+4 S$. If one considers both points to be added in $\mathcal{J}$, then the cost is $12 M+4 S$ (reduced to $11 M+5 S$ by [17]). We will refer to the latter as general addition in $\mathcal{J}$.

In the case of the tripling, Longa and Miri improved the formula proposed by [7] and reduced its cost from $10 M+6 S$ to $6 M+10 S$ in the general case, and to only $7 M+7 S$ if one fixes $a=-3$ [17]. Similarly, an efficient quintupling was presented by the same authors [18 with costs of $10 M+14 S$ in the general case and only $11 M+11 S$ when $a=-3$, improving the formulae by [21] that cost $15 M+10 S$ and $15 M+8 S$ for the corresponding cases.

Variants to the $\mathcal{J}$ have also been proposed. In particular, the four-tuple $\left(X, Y, Z, a Z^{4}\right)$ and five-tuple $\left(X, Y, Z, Z^{2}, Z^{3}\right)$, known as modified Jacobian $\left(\mathcal{J}^{m}\right)$ and Chudnovsky $(\mathcal{C})$ coordinates, respectively, permit to save some operations by passing recurrent values between point operations. Also, a technique that combines different representations (known as mixed coordinates) to yield efficient schemes for the scalar multiplication including precomputation was presented by [5. We discuss the application of these mixed representations in Section 4 . The reader is referred to 15 , for further details.

\section{Composite Operations $d P+Q$}

Our strategy to yield cheaper composite operations of the form $d P+Q$ is based on the efficient use of the new addition formula with identical z-coordinate introduced by Meloni [19, which is described in the following.

Let $P=\left(X_{1}, Y_{1}, Z\right)$ and $Q=\left(X_{2}, Y_{2}, Z\right)$ be two points with the same $z$ coordinates in $\mathcal{J}$ on the elliptic curve $E$. The addition $P+Q=\left(X_{3}, Y_{3}, Z_{3}\right)$ can be obtained as follows:

$$
\begin{aligned}
X_{3} & =\left(Y_{2}-Y_{1}\right)^{2}-\left(X_{2}-X_{1}\right)^{3}-2 X_{1}\left(X_{2}-X_{1}\right)^{2} \\
Y_{3} & =\left(Y_{2}-Y_{1}\right)\left(X_{1}\left(X_{2}-X_{1}\right)^{2}-X_{3}\right)-Y_{1}\left(X_{2}-X_{1}\right)^{3} \\
Z_{3} & =Z\left(X_{2}-X_{1}\right) .
\end{aligned}
$$

This new addition only costs $5 M+2 S$, which represents a significant reduction in comparison with $7 M+4 S$ corresponding to the mixed Jacobian-affine addition. Sadly, it is not possible to directly replace traditional additions with this special operation since, obviously, it is expected that additions are computed over operands with different $z$ coordinates during the scalar multiplication.

The author in [19] applied his formula to the context of scalar multiplication with star addition chains, where the particular sequence of operations allows the replacement of each traditional addition by (3). However, we noticed that the new addition can in fact be applied to a wider context with traditional scalar multiplication methods. In the following, we develop faster composite operations by exploiting the advantages of this special addition on ECC using generic scalar multiplications over prime fields. 


\subsection{Our Methodology}

We propose to compute $d P+Q$ as follows:

$$
d P+Q=P+\ldots+P+P+(P+Q),
$$

where $d$ is a small integer $\geq 2$, and $P, Q$ are points in $\mathcal{J}$ and $\mathcal{A}$ on $E\left(\mathbb{F}_{p}\right)$, respectively.

Strategy (4) would lead to high costs if computed with mixed and general additions. However, we will show in the following that only the first addition in parentheses needs to be computed with a mixed addition. Then, every extra addition can be computed with (3).

First, we compute $P+Q=\left(X_{1}, Y_{1}, Z_{1}\right)+\left(X_{2}, Y_{2}\right)=\left(X_{3}, Y_{3}, Z_{3}\right)$ as mixed Jacobian-affine addition with the following [17:

$$
\begin{aligned}
X_{3} & =4\left(Z_{1}^{3} Y_{2}-Y_{1}\right)^{2}-4\left(Z_{1}^{2} X_{2}-X_{1}\right)^{3}-8 X_{1}\left(Z_{1}^{2} X_{2}-X_{1}\right)^{2} \\
Y_{3} & =2\left(Z_{1}^{3} Y_{2}-Y_{1}\right)\left(4 X_{1}\left(Z_{1}^{2} X_{2}-X_{1}\right)^{2}-X_{3}\right)-8 Y_{1}\left(Z_{1}^{2} X_{2}-X_{1}\right)^{3} \\
Z_{3} & =2 Z_{1}\left(Z_{1}^{2} X_{2}-X_{1}\right)=\left(Z_{1}+Z_{1}^{2} X_{2}-X_{1}\right)^{2}-Z_{1}^{2}-\left(Z_{1}^{2} X_{2}-X_{1}\right)^{2} .
\end{aligned}
$$

The main observation from (5) is that if we assume the next new representation for $P$ :

$$
\left(X_{1}^{(1)}, Y_{1}^{(1)}, Z_{1}^{(1)}\right)=\left(4 X_{1}\left(Z_{1}^{2} X_{2}-X_{1}\right)^{2}, 8 Y_{1}\left(Z_{1}^{2} X_{2}-X_{1}\right)^{3}, 2 Z_{1}\left(Z_{1}^{2} X_{2}-X_{1}\right)\right) \equiv\left(X_{1}, Y_{1}, Z_{1}\right)
$$

we can use the special addition (3) to perform the next addition between $P$ and $(P+Q)$ because both points would have the same $z$ coordinate. It is important to note that the equivalence relation in (6) holds by fixing $\lambda=Z_{1}^{2} X_{2}-X_{1}$ in the equivalence class for $\mathcal{J}$ given in (2). Most importantly, the equivalent point $\left(X_{1}^{(1)}, Y_{1}^{(1)}, Z_{1}^{(1)}\right)$ does not require any extra computation because its coordinates have already been computed in (5).

Similarly, every extra addition with $P$ according to (4) can be performed with the special addition (3) as $P$ always has an equivalent point with the same $\mathrm{z}$ coordinate as the resultant point of the previous computation. In fact, we observe that every addition outside the parentheses in (4) adjusts to the next generic formulae for $j=1$ to $(d-1)$ :

$$
\begin{gathered}
P+(\underbrace{P+\ldots+P}_{(j-1) \text {-terms }}+(P+Q))=\left(X_{1}^{(j)}, Y_{1}^{(j)}, Z_{1}^{(j)}\right)+\left(X_{j+2}, Y_{j+2}, Z_{j+2}\right)=\left(X_{j+3}, Y_{j+3}, Z_{j+3}\right): \\
X_{j+3}=\left(Y_{j+2}-Y_{1}^{(j)}\right)^{2}-\left(X_{j+2}-X_{1}^{(j)}\right)^{3}-2 X_{1}^{(j)}\left(X_{j+2}-X_{1}^{(j)}\right)^{2} \\
Y_{j+3}=\left(Y_{j+2}-Y_{1}^{(j)}\right)\left(X_{1}^{(j)}\left(X_{j+2}-X_{1}^{(j)}\right)^{2}-X_{j+3}\right)-Y_{1}^{(j)}\left(X_{j+2}-X_{1}^{(j)}\right)^{3} \\
Z_{j+3}=Z_{1}^{(j)}\left(X_{j+2}-X_{1}^{(j)}\right) .
\end{gathered}
$$

where $\left(X_{1}^{(j)}, Y_{1}^{(j)}, Z_{1}^{(j)}\right)$ denotes the equivalent point to $P$ for the $j^{\text {th }}$ addition.

As we can see in (7) it holds true that one always gets an equivalent point to $P$ for the following addition by fixing:

$$
\left(X_{1}^{(j+1)}, Y_{1}^{(j+1)}, Z_{1}^{(j+1)}\right)=\left(X_{1}^{(j)}\left(X_{j+2}-X_{1}^{(j)}\right)^{2}, Y_{1}^{(j)}\left(X_{j+2}-X_{1}^{(j)}\right)^{3}, Z_{1}^{(j)}\left(X_{j+2}-X_{1}^{(j)}\right)\right)
$$


which is equivalent to $\left(X_{1}^{(j)}, Y_{1}^{(j)}, Z_{1}^{(j)}\right)$ according to (2), and has the same $z$ coordinate as (7).

The cost of (4) is given by $1 A+(d-1) A^{\prime}$, where $d \in \mathbb{Z}^{+}, d \geq 2$, and $A$ and $A^{\prime}$ denote the cost of the mixed and special additions, respectively. Thus, strategy (4) would cost $(7 M+4 S)+(d-1)(5 M+2 S)$. However, in the following section we will show that by merging the mixed addition between parentheses (see (44)) with the first special addition it is possible to achieve additional savings.

\section{Unified Doubling-Addition (DA) Operation}

When $d=2$, the strategy (41) can be used to perform a doubling-addition (DA) operation as $P+(P+Q)$. We can reduce further the cost of this operation by unifying the first two point additions (i.e., mixed and special additions) into the following unified DA formulae:

$$
X_{4}=\omega^{2}-\theta^{3}-2 X_{1}^{(1)} \theta^{2}, Y_{4}=\omega\left(X_{1}^{(1)} \theta^{2}-X_{4}\right)-Y_{1}^{(1)} \theta^{3}, Z_{4}=Z_{1}^{(1)} \theta .
$$

Where: $\alpha=Z_{1}^{3} Y_{2}-Y_{1}, \beta=Z_{1}^{2} X_{2}-X_{1}$,

$$
\begin{aligned}
X_{1}^{(1)} & =4 X_{1} \beta^{2}, Y_{1}^{(1)}=8 Y_{1} \beta^{3}, Z_{1}^{(1)}=\left(Z_{1}+\beta\right)^{2}-Z_{1}^{2}-\beta^{2}, \\
\theta & =X_{3}-X_{1}^{(1)}=4\left[\alpha^{2}-\beta^{3}-3 X_{1} \beta^{2}\right], \\
\omega & =Y_{3}-Y_{1}^{(1)}=\alpha^{2}+\theta^{2}-(\alpha+\theta)^{2}-16 Y_{1} \beta^{3} .
\end{aligned}
$$

Note that we directly compute $\theta=X_{3}-X_{1}^{(1)}$ and $\omega=Y_{3}-Y_{1}^{(1)}$ to avoid the intermediate computations of $X_{3}, Y_{3}$ and $Z_{3}$ from the first addition (5), saving some field additions and trading one multiplication for one squaring. Thus, the cost of the unified DA is fixed at only $(6 M+5 S)+(5 M+2 S)=11 M+7 S$.

Based on this formula, we can now define the total cost of our methodology for computing composite operations of the form $d P+Q$.

Using (8) to perform the mixed addition and the first special addition, the methodology (4) costs:

$$
(6 M+5 S)+(d-1)(5 M+2 S) .
$$

where $d \geq 2 \in \mathbb{Z}^{+}$for a composite operation of the form $d P+Q$.

Note that, after executing the DA operation as $P+(P+Q)$, the procedure described in Section 3.1 still applies. Hence, the cost of a special addition (i.e., $5 M+2 S$ ) is added at every extra addition with $P$ in (4).

Let us now compare the cost of the methodology (4) with previous formulae. For instance, when $d=2$, (4) computes the DA operation with a cost of $11 M+7 S$, which is superior to the traditional execution consisting of a doubling followed by a mixed addition: $12 M+7 S$ if $a=-3$. In this case, the proposed DA reduces the cost in one multiplication. The new operation is even superior to the improved formulas by [17]: $(3 M+5 S)+(7 M+4 S)=10 M+9 S$, trading one multiplication for two squarings. 
Remarkably, because our strategy does not involve a traditional doubling, the same aforementioned cost for DA is achieved when the parameter $a$ in (11) is randomly chosen. In contrast, a general doubling followed by a mixed addition costs $12 M+9 S$, or $(2 M+8 S)+(7 M+4 S)=9 M+12 S$ with the formulas by 17. In this case, the new DA reduces the cost in one multiplication and two squarings (or trades two multiplications for five squarings, in the second case).

We remark that $2 P+Q$ is a recurrent operation in efficient scalar multiplications. Thus, the new DA can be used to speed up well-known methods such as NAF, wNAF and the Shamir's trick [12] by directly replacing every doubling followed by a mixed addition.

Also, it is important to remark that, as expected, adding one point $P$ at a time in the methodology (4) results efficient for small values of $d$, specifically when $d=2$ and 3. For higher values of $d$ it is better to take advantage of the already efficient doubling, tripling and quintupling operations. In this case, we propose to first perform some computation on the point $P$ using these operations and then apply our approach to the result. For instance, for $d=4,6,7$ and 8 , $d P+Q$ would be computed as follows:

- $4 P+Q=2 P+(2 P+Q)$, which involves a point doubling followed by a DA.

- $6 P+Q=3 P+(3 P+Q)$, which involves a point tripling followed by a DA.

- $7 P+Q=P+(3 P+(3 P+Q))$, which involves a point tripling followed by DA and a general addition.

- $8 P+Q=4 P+(4 P+Q)$, which involves two point doublings followed by DA.

\subsection{Performance Comparison}

Cost estimates using our strategy (4) and the traditional formulae for different composite operations of the form $d P+Q$ are summarized in Table 1 Since we could not find in the literature any effort to accelerate composite operations of the form $d P+Q$ in the case of projective (Jacobian) coordinates over prime fields, new composite operations are compared against operations combining the fastest point operations of form $d P$ (i.e., improved doubling, tripling and quintupling by [17 18]) with addition, in the most efficient way. Thus, $2 P+Q, 3 P+Q$ and $5 Q+P$ are computed by a doubling, tripling and quintupling, respectively, followed by a mixed addition; $4 P+Q$ and $8 P+Q$, by two and three consecutive doublings, respectively, and a mixed addition; $6 P+Q$, by one doubling, one tripling and one mixed addition; and $7 P+Q$, by three doublings, one general addition (with $-P$ ) and one mixed addition. Note that approaches in Table1 1 have been slightly improved for the general case by saving some operations during computation of consecutive doublings $(d=4,8)$, as detailed in Section 2. Also, for the general case, $d=6$, we have reduced the cost further by saving two squarings during computation of a doubling followed by a tripling (see details in Appendix A).

In the case of the proposed composite operations, we show performance when applying the methodology (41) in cases $d=2,3$ and 5, whose cost is given by (9) as detailed in Section 3.1. For $d=4,6,7$ and 8, we use the already efficient 
Table 1. Performance of proposed composite operations of the form $d P+Q$ in comparison with previous formulae. (a) Parameter $a$ is fixed to -3 , (b) Using tripling [17, (c) Using quintupling by [18.

\begin{tabular}{|c|c|c|c|c|c|}
\hline Method & $2 P+Q$ & $3 P+Q$ & $4 P+Q$ & $5 P+Q$ & $6 P+Q$ \\
\hline Ours (5) & $11 M+7 S$ & $16 M+9 S$ & $14 M+12 S^{(\mathrm{a})}$ & $26 M+13 S$ & $18 M+14 S^{(\mathrm{a}, \mathrm{b})}$ \\
& & & $13 M+15 S$ & & $17 M+17 S^{(\mathrm{b})}$ \\
\hline Previous & $10 M+9 S^{(\mathrm{a})}$ & $14 M+11 S^{(\mathrm{a}, \mathrm{b})}$ & $13 M+14 S^{(\mathrm{a})}$ & $18 M+15 S^{(\mathrm{a}, \mathrm{c})}$ & $17 M+16 S^{(\mathrm{a})}$ \\
17]18 & $9 M+12 S$ & $13 M+14 S^{(\mathrm{b})}$ & $13 M+16 S$ & $17 M+18 S^{(\mathrm{c})}$ & $16 M+19 S$ \\
\hline
\end{tabular}

\begin{tabular}{|c|c|c|}
\hline Method & $7 P+Q$ & $8 P+Q$ \\
\hline Ours (5) & $29 M+19 S^{(\mathrm{a})}$ & $17 M+17 S^{(\mathrm{a})}$ \\
& $28 M+22 S$ & $16 M+20 S$ \\
\hline Previous & $28 M+21 S^{(\mathrm{a})}$ & $16 M+19 S^{(\mathrm{a})}$ \\
$17[18$ & $27 M+24 S$ & $16 M+21 S$ \\
\hline
\end{tabular}

DA in combination with the fast doubling, tripling or quintupling by [17 18], as described in Section 3.1.

As we can see, our methodology reduces costs in comparison with the best implementations using previous operation formulae. The only exception is when $d=3$ (special case) or 5 , where the efficient tripling and quintupling formulas previously presented in [17] and [18, respectively, permit to achieve the lowest costs. In most frequent scenario, our new composite operations introduce some savings by trading one multiplication for two squarings (special case, $d=2,4$, 8 ; both cases, $d=6,7)$. In other cases, we trade up to five squarings for only two multiplications (general case, $d=2$ ), or save one squaring (general case, $d=4$, $8)$. The reader must note that the savings are more dramatic if we compare the presented cases with the traditional formulae [12] or the composite operations by [7/21].

\section{New Method for Precomputation}

Precomputed points are extensively used to accelerate the scalar multiplication in applications where extra memory is available. Well-known methods in this category are $w \mathrm{NAF}$ and Frac- $w \mathrm{NAF}$, which rely on precomputations to reduce the Hamming weight of the binary expansion of the scalar, and thus, reduce the cost of the scalar multiplication. In particular, Frac- $w$ NAF requires building the following table with digits $d_{i}[22]$ :

$$
d_{i} \in D_{i}=\{1,3,5, \ldots, m\} .
$$

Using the digit set (10), the average non-zero density $\mathcal{D}$ for Frac-wNAF is [22]:

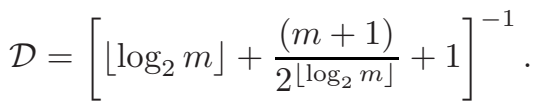


It is important to remark that Frac- $w \mathrm{NAF}$ is a generalization of $w \mathrm{NAF}$ and covers all the possibilities in terms of memory requirements.

We propose a variation to strategy (44) and compute the precomputed table as follows:

$$
d_{i} P=\ldots+2 P+2 P+2 P+P .
$$

We will show that all the additions in (12) can be computed with the special addition with identical $z$-coordinate (3), reducing costs in comparison with previous approaches. Further, some values computed during the mentioned additions are efficiently exploited to minimize costs. In this regard, we present two schemes with different memory requirements that achieve high performance. For the remainder of this work, we refer to them as Schemes 1 and 2.

Our method can be summarized in the following two steps.

\section{Step 1: Computation of precomputed points in Jacobian coordinates}

Point $P$ is assumed to be originally in $\mathcal{A}$. Thus, if we want to use the special addition, we should translate computations to $\mathcal{J}$.

By applying the mixed coordinates approach proposed in [5], we can compute the doubling $2 P$ in $(12)$ in $\mathcal{A}$ and yield the result in $\mathcal{J}$ as follows:

$$
X_{2}=\left(3 x_{1}^{2}+a\right)^{2}-2 \alpha, Y_{2}=\left(3 x_{1}^{2}+a\right)\left(\alpha-X_{2}\right)-8 y_{1}^{4}, Z_{2}=2 y_{1}
$$

with $\alpha=4 x_{1} y_{1}^{2}=2\left[\left(x_{1}+y_{1}^{2}\right)^{2}-x_{1}^{2}-y_{1}^{2}\right]$, where the input and result are $P=\left(x_{1}, y_{1}\right)$ and $2 P=\left(X_{2}, Y_{2}, Z_{2}\right)$, respectively.

Formula (13) is easily derived from the doubling formula in $\mathcal{A}[12$ by applying (2) with $\lambda=2 y_{1}$, and has a cost of only $1 M+5 S$. Note that we have reduced the cost of (13) by replacing the multiplication $4 x_{1} \cdot y_{1}^{2}$ by one squaring and other cheaper operations.

Then, by fixing $\lambda=2 y_{1}$ in (2) we can assume the following equivalent point to $P$ :

$$
P^{(1)}=\left(X_{1}^{(1)}, Y_{1}^{(1)}, Z_{1}^{(1)}\right)=\left(4 x_{1} y_{1}^{2}, 8 y_{1}^{4}, 2 y_{1}\right) \equiv\left(X_{1}, Y_{1}, Z_{1}\right),
$$

which does not introduce extra costs since its coordinates have already been computed in (13). Following additions to compute digits $d_{i}$ would be performed using (4) as follows:

$$
\begin{aligned}
& 1^{\text {st }} 3 P=2 P+P^{(1)}=\left(X_{2}, Y_{2}, Z_{2}\right)+\left(X_{1}^{(1)}, Y_{1}^{(1)}, Z_{1}^{(1)}\right)=\left(X_{3}, Y_{3}, Z_{3}\right): \\
& X_{3}=\left(Y_{1}^{(1)}-Y_{2}\right)^{2}-\left(X_{1}^{(1)}-X_{2}\right)^{3}-2 X_{2}\left(X_{1}^{(1)}-X_{2}\right)^{2} \\
& Y_{3}=\left(Y_{1}^{(1)}-Y_{2}\right)\left(X_{2}\left(X_{1}^{(1)}-X_{2}\right)^{2}-X_{3}\right)-Y_{2}\left(X_{1}^{(1)}-X_{2}\right)^{3} \\
& Z_{3}=Z_{2}\left(X_{1}^{(1)}-X_{2}\right) . \\
& 2^{\text {nd }} \operatorname{Having} 2 P^{(1)}=\left(X_{2}^{(1)}, Y_{2}^{(1)}, Z_{2}^{(1)}\right)=\left(X_{2}\left(X_{1}^{(1)}-X_{2}\right)^{2}, Y_{2}\left(X_{1}^{(1)}-X_{2}\right)^{3}, Z_{2}\left(X_{1}^{(1)}-X_{2}\right)\right) \equiv \\
& \left(X_{2}, Y_{2}, Z_{2}\right), \\
& 5 P=2 P^{(1)}+3 P=\left(X_{2}^{(1)}, Y_{2}^{(1)}, Z_{2}^{(1)}\right)+\left(X_{3}, Y_{3}, Z_{3}\right)=\left(X_{4}, Y_{4}, Z_{4}\right): \\
& X_{4}=\left(Y_{3}-Y_{2}^{(1)}\right)^{2}-\left(X_{3}-X_{2}^{(1)}\right)^{3}-2 X_{2}^{(1)}\left(X_{3}-X_{2}^{(1)}\right)^{2}
\end{aligned}
$$




$$
\begin{aligned}
& Y_{4}=\left(Y_{3}-Y_{2}^{(1)}\right)\left(X_{2}^{(1)}\left(X_{3}-X_{2}^{(1)}\right)^{2}-X_{4}\right)-Y_{2}^{(1)}\left(X_{3}-X_{2}^{(1)}\right)^{3} \\
& Z_{4}=Z_{2}^{(1)}\left(X_{3}-X_{2}^{(1)}\right), A_{4}=\left(X_{3}-X_{2}^{(1)}\right), B_{4}=\left(X_{3}-X_{2}^{(1)}\right)^{2}, C_{4}=\left(X_{3}-X_{2}^{(1)}\right)^{3}
\end{aligned}
$$

$((m-1) / 2)^{t h}$ Having $2 P^{((m-3) / 2)}=\left(X_{2}^{((m-3) / 2)}, Y_{2}^{((m-3) / 2)}, Z_{2}^{((m-3) / 2)}\right)=\left(X_{2}^{((m-5) / 2)}\left(X_{(m-1) / 2}-\right.\right.$ $\left.\left.X_{2}^{((m-5) / 2)}\right)^{2}, \ldots, Y_{2}^{((m-5) / 2)}\left(X_{(m-1) / 2}-X_{2}^{((m-5) / 2)}\right)^{3}, Z_{2}^{((m-5) / 2)}\left(X_{(m-1) / 2}-X_{2}^{((m-5) / 2)}\right)\right) \equiv$ $\left(X_{2}^{((m-5) / 2)}, Y_{2}^{((m-5) / 2)}, Z_{2}^{((m-5) / 2)}\right)$,

$m P=2 P^{((m-3) / 2)}+(m-2) P=\left(X_{2}^{((m-3) / 2)}, Y_{2}^{((m-3) / 2)}, Z_{2}^{((m-3) / 2)}\right)+\left(X_{(m+1) / 2}, Y_{(m+1) / 2}, Z_{(m+1) / 2}\right)$ $m P=2 P^{((m-3) / 2)}+(m-2) P=\left(X_{(m+3) / 2}, Y_{(m+3) / 2}, Z_{(m+3) / 2}\right):$

$X_{(m+3) / 2}=\left(Y_{(m+1) / 2}-Y_{2}^{((m-3) / 2)}\right)^{2}-\left(X_{(m+1) / 2}-X_{2}^{((m-3) / 2)}\right)^{3}-2 X_{2}^{((m-3) / 2)}\left(X_{(m+1) / 2}-\right.$ $\left.X_{2}^{((m-3) / 2)}\right)^{2}$,

$Y_{(m+3) / 2}=\left(Y_{(m+1) / 2}-Y_{2}^{((m-3) / 2)}\right)\left(X_{2}^{(m-3) / 2}\left(X_{(m+1) / 2}-X_{2}^{((m-3) / 2)}\right)^{2}-X_{((m-3) / 2)}\right)-$ $Y_{2}^{(m-3) / 2}\left(X_{(m+1) / 2}-X_{2}^{((m-3) / 2)}\right)^{3}$,

$Z_{(m+3) / 2}=Z_{2}^{(m-3) / 2}\left(X_{(m+1) / 2}-X_{2}^{((m-3) / 2)}\right)$,

$A_{(m+3) / 2}=\left(X_{(m+1) / 2}-X_{2}^{((m-3) / 2)}\right), B_{(m+3) / 2}=\left(X_{(m+1) / 2}-X_{2}^{((m-3) / 2)}\right)^{2}, C_{(m+3) / 2}=$ $\left(X_{(m+1) / 2}-X_{2}^{((m-3) / 2)}\right)^{3}$.

Values $A_{i}$ and $\left(B_{i}, C_{i}\right)$, for $i=4$ to $(m+3) / 2$, are stored for Schemes 1 and 2 , respectively, and used in Step 2 to save some computations when converting points to $\mathcal{A}$.

Step 2: Conversion to affine coordinates

Points $\left(X_{i}, Y_{i}, Z_{i}\right)$ from Step 1 , for $i$ from 3 to $(m+3) / 2$, have to be converted back to $\mathcal{A}$ since this would allow the use of the efficient mixed addition during the scalar multiplication. This can be achieved by means of the following:

$$
\left(X_{i} / Z_{i}^{2}, Y_{i} / Z_{i}^{3}, 1\right)
$$

To avoid the computation of several expensive inversions when using (15) for each point in the case $m>3$ (10), we use the method due to Montgomery, called simultaneous inversion [12, to limit the requirement to only one inversion.

In Scheme 1, we first compute the inverse $r=Z_{(m+3) / 2}^{-1}$, and then recover every point using (15) as follows:

$$
\begin{aligned}
& m P: x_{(m+3) / 2}=r^{2} \cdot X_{(m+3) / 2}, y_{(m+3) / 2}=r^{3} \cdot Y_{(m+3) / 2} \\
& (m-2) P: r=r \cdot A_{(m+3) / 2}, x_{(m+1) / 2}=r^{2} \cdot X_{(m+1) / 2}, y_{(m+1) / 2}=r^{3} \cdot Y_{(m+1) / 2} \\
& \vdots \\
& 3 P: r=r . A_{4}, x_{3}=r^{2} \cdot X_{3}, y_{3}=r^{3} \cdot Y_{3} .
\end{aligned}
$$


It is important to observe that $Z_{j}=Z_{3} \times \prod_{i=4}^{j} A_{i}$ for $j=4$ to $(m+3) / 2$, according to Step 1 , and hence, for $i=(m-2)$ down to $3, Z_{(i+3) / 2}^{-1}$ for each point $i P$ is recovered at every multiplication $r \cdot A_{(i+5) / 2}$.

For Scheme 2, we first compute $r_{1}=\left(Z_{(m+3) / 2}^{-1}\right)^{2}$ and $r_{2}=\left(Z_{(m+3) / 2}^{-1}\right)^{3}$, and then recover every point using (15) as follows:

$m P: x_{(m+3) / 2}=r_{1} \cdot X_{(m+3) / 2}, y_{(m+3) / 2}=r_{2} \cdot Y_{(m+3) / 2}$

$(m-2) P: r_{1}=r_{1} \cdot B_{(m+3) / 2}, r_{2}=r_{2} \cdot C_{(m+3) / 2}, x_{(m+1) / 2}=r_{1} \cdot X_{(m+1) / 2}, y_{(m+1) / 2}=$ $r_{2} \cdot Y_{(m+1) / 2}$

:

$3 P: r_{1}=r_{1} \cdot B_{4}, r_{2}=r_{2} \cdot C_{4}, x_{3}=r_{1} \cdot X_{3}, y_{3}=r_{2} \cdot Y_{3}$.

In this case $Z_{j}^{2}=Z_{3}^{2} \times \prod_{i=4}^{j} B_{i}$ and $Z_{j}^{3}=Z_{3}^{3} \times \prod_{i=4}^{j} C_{i}$ for $j=4$ to $(m+$ $3) / 2$, according to Step 1, and hence, for $i=(m-2)$ down to 3, the pair $\left(Z_{(i+3) / 2}^{-2}, Z_{(i+3) / 2}^{-3}\right)$ for each point $i P$ is recovered at every multiplication $r_{1}$. $B_{(i+5) / 2}$ and $r_{2} \cdot C_{(i+5) / 2}$.

\subsection{Cost Analysis}

In total, Scheme 1 has the following cost when computing the precomputed table (10):

$$
\text { Cost }_{\text {Scheme } 1}=1 I+(9 L) M+(3 L+5) S \text {, }
$$

where $L=(m-1) / 2$ represents the number of points. In terms of memory usage, Scheme 1 requires $(3 L+3)$ registers for temporary calculations and storing the precomputed points. We will show later that this requirement does not exceed the number of available registers for the scalar multiplication for practical values of $L$.

In the case of Scheme 2, the cost is as follows:

$$
{ }^{\text {Cost }} \text { Scheme } 2=1 I+(9 L) M+(2 L+6) S .
$$

For this scheme, we require $(4 L+1)$ registers when $L>1$. For $L=1$, the requirement is fixed at 6 registers. It will be shown that this requirement does not exceed the memory allocated for scalar multiplication for small values of $L$. For a detailed description of the estimation of costs and memory requirements for Schemes 1/2, we refer to Appendix B.

As we can see from (16) and (17), Scheme 2 reduces further the cost to compute the precomputed table at the expense of some extra memory. In the following, we analyze the memory requirements for the scalar multiplication and determine if our method adjusts to such constrains.

Considering that the precomputed table requires $2 L$ registers for storing $L$ points, the total requirement of the scalar multiplication is given by $(2 L+R)$ registers, where $R$ is the number of registers needed by the most memory-consuming point operation in a given implementation. On scalar multiplications using solely radix 2 , addition is usually such operation. Depending on the used coordinates 
and/or implementation details, point addition can require from $7 / 8$ registers in $\mathcal{J}$ 17] and $\mathcal{J}^{m}$, respectively, to 8 registers for an SSCA-protected version [20]. If the scalar multiplication includes radix 3 in its expansion, then tripling becomes the most expensive operation with a requirement of up to $9 / 10$ registers 7[17. Consequently, Scheme 2 adjusts to the previous requirements for small precomputed tables with $L=1$ to 3 if addition is the main operation. If we also consider tripling, Scheme 2 is suitable for values $L=1$ to 4 . In the case of Scheme 1, it follows the memory constrains for values $L=1$ to 5 and $L=1$ to 7 for radix- 2 and radix-3 cases, respectively, which demonstrates that this scheme is efficient for practical Frac- $w$ NAF implementations. The reader must note that, in general, values $L>7$ are not efficient since the cost of computing the precomputed table results more expensive than the savings achieved by precomputation during the scalar multiplication.

In the following section, we analyze the performance of our method in comparison with previous efforts.

\subsection{Performance Comparison}

There are different efficient schemes to compute precomputed points in the literature. The simplest approaches suggest performing computations in $\mathcal{A}$ or $\mathcal{C}$ using the chain $P \rightarrow 3 P \rightarrow 5 P \rightarrow \ldots \rightarrow m P$. The latter requires one doubling and $L=(m-1) / 2$ additions, which can be expressed as follows in terms of field operations for the mentioned cases:

$$
\begin{aligned}
\operatorname{Cost}_{\mathcal{A}} & =(L+1) I+(2 L+2) M+(L+2) S \\
\operatorname{Cost}_{\mathcal{C}} & =(10 L-1) M+(4 L+5) S
\end{aligned}
$$

Note that (19) shows a better performance than the estimated cost given by 6 . since we are considering that the doubling $2 P$ is computed as $2 \mathcal{A} \rightarrow \mathcal{C}$ with a cost of $2 M+5 S$, the first addition $P+2 P$ computed with a mixed addition as $\mathcal{A}+\mathcal{C} \rightarrow \mathcal{C}(7 M+4 S)$, and the following $(L-1)$ additions as $\mathcal{C}+\mathcal{C} \rightarrow \mathcal{C}(10 M+4 S)$. The new operation costs are obtained by applying the technique of replacing multiplications by squarings introduced in 17. The memory requirements of the $\mathcal{A}$ - and $\mathcal{C}$-based methods are $2 L+R$ and $5 L+R$ registers, respectively.

Other methods that achieve better performance in scenarios where inversion is relatively expensive, perform computations in Projective $\mathcal{P}, \mathcal{J}$ or $\mathcal{C}$ coordinates and, then, convert the points to $\mathcal{A}$ by using the Montgomery's method to reduce the number of required inversions to only one. Cost for these methods are shown in the following [6], considering the general assumption $1 S \approx 0.8 M$ :

$$
\begin{aligned}
\operatorname{Cost}_{\mathcal{P} \rightarrow \mathcal{A}} & =1 I+(16 L-3) M+(3 L+5) S=1 I+(18.4 L+1) M \\
\operatorname{Cost}_{\mathcal{J} \rightarrow \mathcal{A}} & =1 I+(16 L-5) M+(5 L+5) S=1 I+(20 L-1) M \\
\operatorname{Cost}_{\mathcal{C} \rightarrow \mathcal{A}} & =1 I+(16 L-4) M+(5 L-5) S=1 I+(20 L) M .
\end{aligned}
$$

Recently, Dahmen et al. 6] proposed a new scheme, whose computations were efficiently performed using solely formulae in $\mathcal{A}$. Also, the number of inversions 
was limited to only one by means on the Montgomery's method. This scheme costs:

$$
\operatorname{Cost}_{[6]}=1 I+(10 L-1) M+(4 L+4) S=1 I+(13.2 L+2.2) M,
$$

that shows its superiority when compared with all the previous methods requiring only one inversion. However, our method achieves even lower costs as shown in Section 4.1:

$$
\text { Cost }_{\text {Scheme } 1}=1 I+(11.4 L+4) M, \text { Cost }_{\text {Scheme } 2}=1 I+(10.6 L+4.8) M \text {. }
$$

which make our approach, and specifically Scheme 2, the fastest in the literature when the number of inversions is limited to one.

For comparing with the approach (18), which includes several field inversions in their computation, it is better to specify the range of $I / M$ ratios for which each method is superior. Table 2 shows the $I / M$ values for which our schemes and the $\mathcal{A}$-based scheme are the most efficient for a given number of precomputed points. To present a fair comparison, methods are compared according to the memory constrains for the scalar multiplication using radix-2. As analyzed in Section 4.1, Schemes 1 and 2 are suitable for values $L=1$ to 5 and $L=1$ to 4 , respectively.

Table 2. $I / M$ ranges for which each method achieves the lowest cost

\begin{tabular}{|c|c|c|c|c|c|}
\hline \# Points & 1 & 2 & 3 & 4 & 5 \\
\hline Scheme 1 & - & - & - & $\geq 8.1$ & $\geq 8.7$ \\
Scheme 2 & $\geq 9$ & $\geq 8.4$ & $\geq 8.2$ & - & - \\
Affine (18) & $\leq 9$ & $\leq 8.4$ & $\leq 8.2$ & $\leq 8.1$ & $\leq 8.7$ \\
\hline
\end{tabular}

As it can be seen, our schemes outperform the $\mathcal{A}$-based approach for the most commonly found $I / M$ ratios, where inversion is relatively expensive. In average, Schemes 1 and 2 are superior when inversion is more than 9 times the cost of multiplication. As discussed in Section 2, it is usually expected that $I / M>30$.

Finally, we compare performance of our schemes with the $\mathcal{C}$-based approach, whose cost is given by (19). In this case, we should also consider the scalar multiplication cost in our comparisons since precomputations in $\mathcal{C}$ require different computing and memory requirements to the $\mathcal{A}$ case. When precomputations are in $\mathcal{C}$, 5 proposed the use of $\mathcal{J}+\mathcal{C} \rightarrow \mathcal{J}^{m}$ to perform additions $(10 M+6 S)$, $2 \mathcal{J}^{m} \rightarrow \mathcal{J}$ to every doubling preceding an addition $(2 M+5 S)$, and $2 \mathcal{J}^{m} \rightarrow \mathcal{J}^{m}$ $(3 M+5 S)$ to the rest of doublings. Note that we have reduced further the cost of the mentioned operations by applying the same technique introduced in [17] to replace multiplications by squarings. Following this scheme, the scalar multiplication cost including precomputations is as follows:

$[n D((10 M+6 S)+(2 M+5 S))+n(1-D)(3 M+5 S)]+[(10 L-1) M+(4 L+5) S]$

where $\mathcal{D}$ represents the Hamming weight as expressed in (11). 
For our approach, we consider an improved scheme taking advantage of the faster DA operation proposed in Section 3.1. Thus, we use $\mathcal{J}+(\mathcal{J}+\mathcal{A}) \rightarrow \mathcal{J}$ to perform doubling-addition operations following the form $P+(P+Q)$ with a cost of $11 M+7 S$, and the fast point operations by [17. With this scheme, the scalar multiplication cost including precomputations is as follows:

$$
[n \mathcal{D}(11 M+7 S)+n(1-\mathcal{D})(3 M+5 S)]+\text { Cost }_{\text {Scheme } 1 / 2} .
$$

We also include in our comparison a traditional scheme using $\mathcal{J}$ and only one inversion to assess the advantages of our improved scheme (25). By using (21) and the point operations by [17, the cost of the scalar multiplication including precomputations is given by:

$$
[n \mathcal{D}(7 M+4 S)+n(3 M+5 S)]+[1 I+(20 L-1) M]
$$

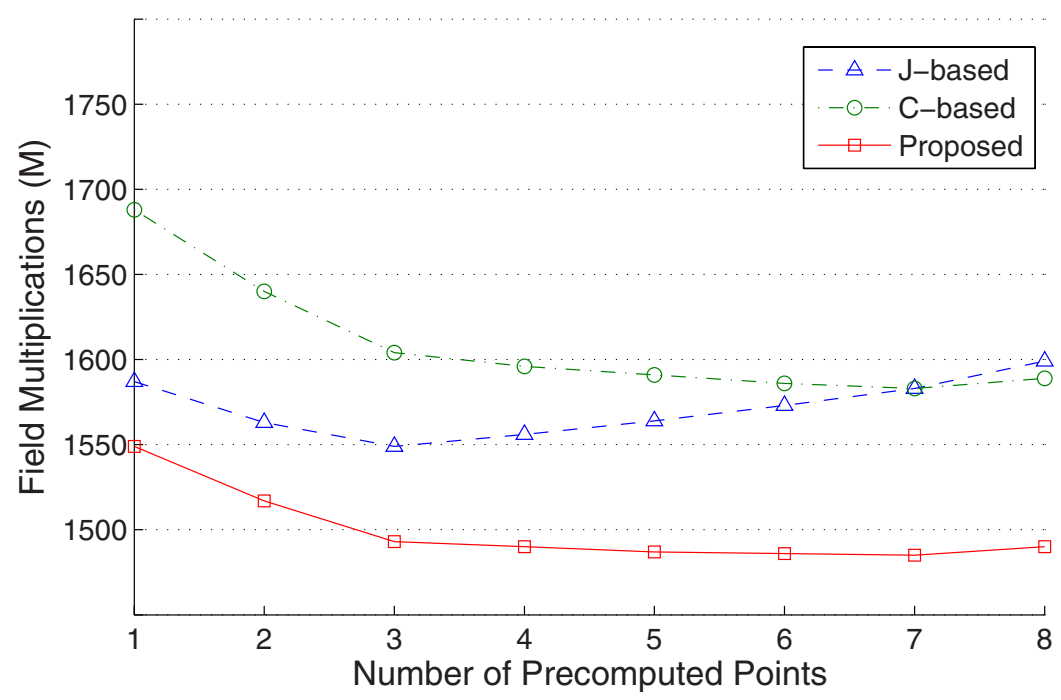

Fig. 1. Cost performance of the proposed scheme and previous methods to perform the scalar multiplication including precomputation $(1 I=30 M, 1 S=0.8 M, n=160$ bits)

Figure 1 plots the costs of our scheme (25), and the $\mathcal{C}-$ and $\mathcal{J}$-based approaches as given by (24) and (26), assuming $1 I=30 M, 1 S=0.8 M$ and $n=160$ bits. As we can see, our proposed scheme outperforms both methods, introducing an improvement of up to $6.2 \%$ in comparison with the already optimized $\mathcal{J}$-based approach, when assuming unrestricted availability of memory (optimal case when using the Frac-wNAF method with 7 precomputed points). We remark that the given estimation is a lower bound as additions and other operations are not included in the cost. The new DA offers a reduced number of these operations, and thus, a real implementation would achieve a higher performance 
improvement. Also, it is important to note that the improvement would be even more significant in implementations where a hardware multiplier executes both squarings and multiplications (i.e., $1 S=1 M)$.

The previous analysis does not take into consideration memory consumption when comparing the $\mathcal{C}$-based approach and our method. Recalling that the former has a memory requirement of $(5 L+R)$ and assuming $R=8$, Table 3 summarizes the $I / M$ break even points at which both methods perform equivalently for a given number of available registers. Similarly, costs have been derived according to (24) and (25). Notice that our method is superior in any case the $I / M$ ratio is below the displayed numbers, which makes it superior for typical $I / M$ ratios, as discussed in Section 2.

Table 3. $I / M$ break even points for which our schemes and the $\mathcal{C}$-based approach perform equivalently for a given number of registers $(n=160 \mathrm{bits})$. (1) Scheme 1 and (2) Scheme 2.

\begin{tabular}{|l|c|c|c|c|c|c|c|}
\hline \# Registers & $\leq 10$ & 12 & 13 & $14-16$ & 17 & $18-20$ & $21-23$ \\
\hline Break point & $337^{(1,2)}$ & $369^{(2)}$ & $201^{(2)}$ & $224^{(2)}$ & $228^{(2)}$ & $180^{(2)}$ & $182^{(2)}$ \\
\hline \# Registers & 24 & $25-28$ & $29-32$ & $33-37$ & $38-42$ & $\geq 43$ & \\
\hline Break point & $144^{(1)}$ & $149^{(2)}$ & $141^{(2)}$ & $136^{(2)}$ & $131^{(2)}$ & $128^{(2)}$ & \\
\hline
\end{tabular}

\section{Conclusions}

We have described an innovative methodology to derive composite operations of the form $d P+Q$ by applying the special addition with identical $z$-coordinate to the setting of generic scalar multiplications over prime fields. These new operations are shown to be faster than operations built on top of previous formulae, which would potentially speed up computations in all known binary methods and in new scalar multiplications using other radices beside 2 such as double-base 7, triple-base 21] or $m b \mathrm{NAF}$ [18].

In the second part of this work, we presented two variants of a new precomputation scheme for window-based scalar multiplications, and showed that our methods offer the lowest costs, given by $1 I+(9 L) M+(3 L+5) S$ and $1 I+(9 L) M+(2 L+6) S$, when using only one inversion. For the rest of cases, we demonstrated that they achieve superior performance for most common $I / M$ ratios found in practical implementations.

\section{References}

1. Avanzi, R., Cohen, H., Doche, C., Frey, G., Lange, T., Nguyen, K., Vercauteren, F.: Handbook of Elliptic and Hyperelliptic Curve Cryptography. CRC Press, Boca Raton (2005)

2. Brown, M., Hankerson, D., Lopez, J., Menezes, A.: Software Implementation of the NIST elliptic curves over prime fields. In: Naccache, D. (ed.) CT-RSA 2001. LNCS, vol. 2020, pp. 250-265. Springer, Heidelberg (2001) 
3. Billet, O., Joye, M.: Fast Point Multiplication on Elliptic Curves through Isogenies. In: Fossorier, M.P.C., Høholdt, T., Poli, A. (eds.) AAECC 2003. LNCS, vol. 2643, pp. 43-50. Springer, Heidelberg (2003)

4. Ciet, M., Joye, M., Lauter, K., Montgomery, P.L.: Trading Inversions for Multiplications in Elliptic Curve Cryptography. Designs, Codes and Cryptography 39(2), 189-206 (2006)

5. Cohen, H., Miyaji, A., Ono, T.: Efficient Elliptic Curve Exponentiation using Mixed Coordinates. In: Ohta, K., Pei, D. (eds.) ASIACRYPT 1998. LNCS, vol. 1514, pp. 51-65. Springer, Heidelberg (1998)

6. Dahmen, E., Okeya, K., Schepers, D.: Affine Precomputation with Sole Inversion in Elliptic Curve Cryptography. In: Pieprzyk, J., Ghodosi, H., Dawson, E. (eds.) ACISP 2007. LNCS, vol. 4586, pp. 245-258. Springer, Heidelberg (2007)

7. Dimitrov, V., Imbert, L., Mishra, P.K.: Efficient and Secure Elliptic Curve Point Multiplication using Double-Base Chains. In: Roy, B. (ed.) ASIACRYPT 2005. LNCS, vol. 3788, pp. 59-78. Springer, Heidelberg (2005)

8. Doche, C., Icart, T., Kohel, D.: Efficient Scalar Multiplication by Isogeny Decompositions. In: Yung, M., Dodis, Y., Kiayias, A., Malkin, T.G. (eds.) PKC 2006. LNCS, vol. 3958, pp. 191-206. Springer, Heidelberg (2006)

9. Eisentraeger, K., Lauter, K., Montgomery, P.: Fast Elliptic Curve Arithmetic and Improved Weil Pairing Evaluation. In: Joye, M. (ed.) CT-RSA 2003. LNCS, vol. 2612, pp. 343-354. Springer, Heidelberg (2003)

10. Elmegaard-Fessel, L.: Efficient Scalar Multiplication and Security against Power Analysis in Cryptosystems based on the NIST Elliptic Curves over Prime Fields, Master Thesis, University of Copenhagen (2006)

11. FIPS PUB 186-2. Digital Signature Standard (DSS). National Institute of Standards and Technology (NIST) (2000)

12. Hankerson, D., Menezes, A., Vanstone, S.: Guide to Elliptic Curve Cryptography. Springer, Heidelberg (2004)

13. IEEE Std 1363-2000. IEEE Standard Specifications for Public-Key Cryptography. The Institute of Electrical and Electronics Engineers (IEEE) (2000)

14. Itoh, K., Takenaka, M., Torii, N., Temma, S., Kurihara, Y.: Fast Implementation of Public-Key Cryptography on a DSP TMS320C6201. In: Koç, Ç.K., Paar, C. (eds.) CHES 1999. LNCS, vol. 1717, pp. 61-72. Springer, Heidelberg (1999)

15. Lim, C.H., Hwang, H.S.: Fast implementation of Elliptic Curve Arithmetic in GF(pn). In: Imai, H., Zheng, Y. (eds.) PKC 2000. LNCS, vol. 1751, pp. 405-421. Springer, Heidelberg (2000)

16. Longa, P.: Accelerating the Scalar Multiplication on Elliptic Curve Cryptosystems over Prime Fields, Master's Thesis, University of Ottawa (June 2007)

17. Longa, P., Miri, A.: Fast and Flexible Elliptic Curve Point Arithmetic over Prime Fields. IEEE Transactions on Computers (to appear, 2007), http://doi.ieeecomputersociety.org/10.1109/TC.2007.70815

18. Longa, P., Miri, A.: New Multibase Non-Adjacent Form Scalar Multiplication and its Application to Elliptic Curve Cryptosystems (submitted, 2007)

19. Meloni, N.: Fast and Secure Elliptic Curve Scalar Multiplication over Prime Fields using Special Addition Chains. Cryptology ePrint Archive, Report 2006/216 (2006)

20. Mishra, P.: Scalar Multiplication in Elliptic Curve Cryptosystems: Pipelining with Precomputations. Cryptology ePrint Archive, Report 2004/191 (2004)

21. Mishra, P., Dimitrov, V.: Efficient Quintuple Formulas for Elliptic Curves and Efficient Scalar Multiplication using Multibase Number Representation. Cryptology ePrint Archive, Report 2007/040 (2007) 
22. Moller, B.: Improved Techniques for Fast Exponentiation. In: Lee, P.J., Lim, C.H. (eds.) ICISC 2002. LNCS, vol. 2587, pp. 298-312. Springer, Heidelberg (2003)

23. Solinas, J.: Generalized Mersenne Numbers, Technical Report CORR-99-39, Dept. of C\&O, University of Waterloo (1999)

24. Takagi, T., Yen, S.-M., Wu, B.-C.: Radix-r Non-Adjacent Form. In: Zhang, K., Zheng, Y. (eds.) ISC 2004. LNCS, vol. 3225, pp. 99-110. Springer, Heidelberg (2004)

\section{A Doubling-Tripling formulae}

Having $P=\left(X_{1}, Y_{1}, Z_{1}\right)$ on the elliptic curve $E$, the doubling $2 P=\left(X_{2}, Y_{2}, Z_{2}\right)$ in Jacobian coordinates is computed by [17:

$$
\begin{gathered}
X_{2}=A^{2}-2 B, Y_{2}=A \cdot\left(B-X_{2}\right)-8 D, Z_{2}=\left(Y_{1}+Z_{1}\right)^{2}-C-E, \\
A=3 G+H, B=2\left[\left(X_{1}+C\right)^{2}-G-D\right], C=Y_{1}^{2}, D=C^{2}, E=Z_{1}^{2}, F=E^{2}, \\
G=X_{1}^{2}, H=a \cdot F,
\end{gathered}
$$

and followed by the next revised tripling formulae to yield $6 P=\left(X_{3}, Y_{3}, Z_{3}\right)$, which derives from the fast tripling in [17]:

$$
\begin{gathered}
X_{3}=I \cdot T+X, Y_{3}=8 Y_{2} \cdot(V-W), Z_{3}=2 Z_{2} \cdot P, \\
I=Y_{2}^{2}, J=I^{2}, K=16 D \cdot H, L=X_{2}^{2}, M=3 L+K, N=P^{2}, \\
P=6\left[\left(X_{1}+I\right)^{2}-L-J\right]-N, R=P^{2}, S=(M+P)^{2}-N-R, T=16 J-S, \\
U=16 J+T, V=-T \cdot U, W=P \cdot R, X=4 X_{2} \cdot R .
\end{gathered}
$$

The general doubling still requires $2 M+8 S$, but the tripling reduces its cost to $7 M+7 S$ by using previously computed values $D$ and $H$ to compute $a Z_{2}^{4}$ as $16 Y_{1}^{4} \cdot a Z_{1}^{4}$. Thus, the total cost of a Doubling-Tripling operation when the parameter $a$ is randomly chosen is $9 M+15 S$.

\section{B Cost Analysis of Precomputation Scheme}

Scheme 1 has the following cost:

$$
\text { Cost }_{\text {Scheme } 1}=1 I+(9 L) M+(3 L+5) S,
$$

and requires $(3 L+3)$ registers, where $L$ is the number of points in the precomputed table.

Proof: The doubling (13) of Step 1, Section 4, cost $1 M+5 S$ and requires 6 temporary registers. The first addition $2 P+P$ in (12) using the special addition with identical $z$-coordinates costs $5 M+2 S$ and requires 6 temporary registers if the precomputed table contains only one point. Otherwise, it would require 6 temporary registers for calculations, and 2 extra registers to store the $(X, Y)$ 
coordinates of $3 P$. Following additions in (12) using the special addition with identical $z$-coordinates cost $5 M+2 S$ per extra point, and 6 temporary registers for calculations, and $3 / 4$ extra registers per each point for Schemes $1 / 2$, respectively to store the values $(X, Y, A, B, C)$. In the last iteration of Step 1 (Section 4$)$ the memory requirement is reduced to storing $(X, Y, B)$ values in temporary registers. Thus, Schemes 1 and 2 only require the previous 6 registers plus 1 extra register in this case. Finally, the modified Montgomery's method corresponding to Step 2 costs $1 I+(3 M+1 S)+(4 M+1 S)(L-1)$ and $1 I+$ $(3 M+1 S)+4 M(L-1)$ for Schemes 1 and 2 respectively, and requires $4 /$ 5 temporary registers for calculations, in addition to registers for storing the affine coordinates $(x, y)$ of the precomputed points. Thus, Steps 1 and 2 cost $(1 M+5 S)+(5 M+2 S)+(5 M+2 S)(L-1)$ and $1 I+(3 M+1 S)+(4 M+1 S)(L-1)$, respectively. By adding these values, we obtain the cost of Scheme 1 as presented above.

Regarding memory requirements, the doubling (13) needs 6 temporary registers $T_{1}, \ldots, T_{6}$. The same registers can be reused by the first special addition. Additionally, it needs 2 extra registers to store $(X, Y)$ coordinates corresponding to $3 P$, making a total of 8 registers. Following additions also reuses temporary registers $T_{1}, \ldots, T_{6}$, and requires 3 registers per point, excepting the last one, to store $(X, Y, A)$ values. For the last iteration, we only require registers $T_{1}, \ldots, T_{6}$ and 1 extra register to store $A$ since the last $(X, Y)$ coordinates are store in $T_{1}$ and $T_{2}$. That makes an accumulated requirement of $6+3(L-1)=3 L+3$ at the end of Step 1, for $L \geq 2$. If $L=1$, we only require the first special addition, fixing the requirement at only 6 registers (note that in this case $(X, Y)$ coordinates are stored in $T_{1}$ and $T_{2}$ ). Step 2 only requires 4 registers for calculations, two of which store the first pair $(x, y)$. The rest of points require $3(L-1)$ registers, making a total requirement of $4+3(L-1)=3 L+1$. In conclusion, Scheme 1 requires $3 L+3$ registers.

The cost and memory estimation for Scheme 2 easily follows. 